\title{
Epigenetic signature of preterm birth in adult twins
}

\author{
Qihua $\operatorname{Tan}^{1,2^{*}}$ (D), Shuxia Li ${ }^{2}$, Morten Frost ${ }^{3}$, Marianne Nygaard', Mette Soerensen ${ }^{1}$, Martin Larsen ${ }^{2,4}$, \\ Kaare Christensen ${ }^{1,2}$ and Lene Christiansen ${ }^{1}$
}

\begin{abstract}
Background: Preterm birth is a leading cause of perinatal mortality and long-term health consequences. Epigenetic mechanisms may have been at play in preterm birth survivors, and these could be persistent and detrimental to health later in life.

Methods: We performed a genome-wide DNA methylation profiling in adult twins of premature birth to identify genomic regions under differential epigenetic regulation in 144 twins with a median age of 33 years (age range 30-36).

Results: Association analysis detected three genomic regions annotated to the SDHAP3, TAGLN3 and GSTT1 genes on chromosomes 5, 3 and 22 (FWER: 0.01, 0.02 and 0.04) respectively. These genes display strong involvement in neurodevelopmental disorders, cancer susceptibility and premature delivery. The three identified significant regions were successfully replicated in an independent sample of twins of even older age (median age 66, range 56-80) with similar regulatory patterns and nominal $p$ values $<5.05 e-04$. Biological pathway analysis detected five significantly enriched pathways all explicitly involved in immune responses.

Conclusion: We have found novel evidence associating premature delivery with epigenetic modification of important genes/pathways and revealed that preterm birth, as an early life event, could be related to differential methylation regulation patterns observable in adults and even at high ages which could potentially mediate susceptibility to agerelated diseases and adult health.
\end{abstract}

Keywords: Preterm birth, Twins, Epigenetics, Epigenome-wide association study, Adults

\section{Background}

Preterm birth (PTB) or premature birth is defined as birth before 37 weeks of pregnancy. With a prevalence estimated from 5 to $18 \%$ in singleton pregnancies across 184 countries according to the World Health Organization and over $50 \%$ in twin pregnancies in the USA [1], PTB is a leading cause of perinatal mortality as well as long-term morbidity and health consequences. Survivors of PTB were subject to adaptive mechanisms that might be deleterious later in life, and are more susceptible to early on-set chronic diseases [2] including cardiovascular disease [3], metabolic disorders [4], respiratory complications [5] and mental and cognitive

\footnotetext{
* Correspondence: qtan@health.sdu.dk

${ }^{1}$ Epidemiology and Biostatistics, Department of Public Health, Faculty of Health Science, University of Southern Denmark, J. B. Winsløws Vej 9B, DK-5000 Odense, Denmark

${ }^{2}$ Unit of Human Genetics, Department of Clinical Research, University of Southern Denmark, Odense, Denmark

Full list of author information is available at the end of the article
}

impairments [6]. Despite the strong epidemiological evidence, the molecular mechanisms and etiology behind these phenotypes have been poorly understood. Preterm infants are exposed to various stressful conditions in the peridelivery period, a critical stage for their organ development. Molecular mechanisms including epigenetic modification may have been involved in the adaptation to adverse environment which, in the long-run, could be detrimental to health [7-9]. It has been hypothesized that epigenetic modifications such as DNA methylation induced by PTB may lead to long-term consequences and increased susceptibility to adult-onset diseases [10-12].

Advantaged by the emerging new technology in genomic analysis of DNA methylation, epigenome-wide association studies (EWAS) have been done to look for DNA methylation markers of PTB in neonates [13-16] and have reported differentially methylated sites implicated in neural function [16], or with increased risk for adverse 
health outcomes later in life [13]. Notably, PTB-associated methylation patterns were also investigated in adolescents by Cruickshank et al. [14] and Simpkin et al. [15] in their longitudinal samples. Although relatively large numbers of CpG sites were found significantly differentially methylated in association with PTB at birth, they are largely resolved in adolescents in both studies. Nevertheless, persistent methylation differences were identified at ten CpG sites in the study by Cruickshank et al. [14] reflecting a lasting epigenetic effect of PTB.

The fact that PTB is associated with an increased risk of chronic diseases in adults suggests that it is of high importance to focus on the epigenetic signature of PTB that mediates the long-term health consequences. Given the high prevalence of PTB in twin pregnancies, epigenetic analysis of PTB in twins is therefore especially important and valuable for the health of the twin population and for the general population as well. Using relatively large numbers of adult twin samples for discovery (144 twins) and replication (350 twins), we conducted an epigenomic profiling of the DNA methylome to look for genomic sites and regions under epigenetic regulation in association with PTB in adult subjects.

\section{Methods}

\section{The discovery samples}

The discovery samples in this study consisted of 72 pairs of identical twins (144 individuals, 78 males and 66 females) aged 30 to 36 years with a median age of 33 (Table 1). Gestational ages were collected from the Danish Medical Birth Registry (DMBR) established in 1973. The median of gestational age was 39 weeks with a minimum of 33 and a maximum of 42 weeks. A total of 26 twins had their gestational ages $<37$ weeks. The samples formed a subset (those born after 1973 when gestational age was

Table 1 Descriptive statistics for the discovery and replication samples

\begin{tabular}{lll}
\hline Variables & Discovery & Replication \\
\hline Age, year & 33 & 66 \\
Median & $30-36$ & $56-80$ \\
Range & 39 & 0 (weeks before term) \\
Gestational age, week & $0-8$ (weeks before term) \\
Median & $33-42$ & Midwife \\
Range & DMBR & \\
Report method & & 192 \\
Sample size & 78 & 158 \\
Male & 66 & 350 \\
Female & 144 & 40 (more than 3 weeks before term) \\
Total & 26 & \\
PTB &
\end{tabular}

DMBR Danish Medical Birth Registry recorded by DMBR) of 150 pairs of identical twins discordant for birthweight used in an EWAS by Tan et al. [17]. Figure 1 displays the samples by plotting individual gestational age against birthweight. There is a moderate correlation of 0.52 between gestational age (PTB indicated by empty spots) and birthweight. Although no significant association was found between birthweight and DNA methylation [17], we adjusted for individual birthweight in all our analyses.

\section{Blood sampling and DNA extraction}

Blood sampling and DNA preparation were described by Tan et al. [17]. In brief, ethylene di-amine tetra acetic acid (EDTA)-anticoagulated blood samples were collected. The blood was centrifuged at $1000 \mathrm{~g}$ for $10 \mathrm{~min}$, and buffy-coat was frozen in aliquots at $-80{ }^{\circ} \mathrm{C}$. DNA was isolated from the buffy-coats using the salt precipitation method applying either a manual protocol or a semi-automated protocol based on the Autopure System (Qiagen, Hilden, Germany). Bisulphite treatment of 500 ng template genomic DNA was carried out with the EZ-96 DNA methylation kit (Zymo Research, Orange County, USA) following the manufacturer's protocol.

\section{DNA methylation data}

Genome-wide DNA methylation was analysed using the Illumina Infinium HumanMethylation450 Beadchip assay (Illumina, San Diego, CA, USA) at Leiden University Medical Center or at GenomeScan B.V., Leiden, The Netherlands. The array interrogates more than 485,000 CpG sites across and beyond gene and CpG island regions in the human genome. The laboratory experiment was conducted according to the array manufacturer's

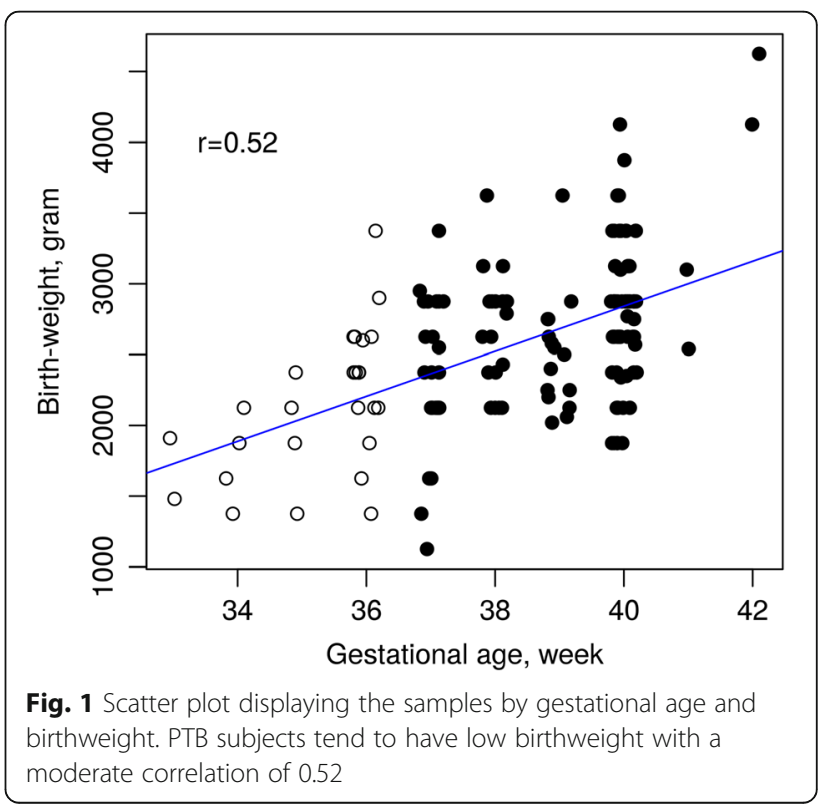


instructions. Twins of each pair were processed together on the same array to minimize batch effect. The quality of DNA methylation data was controlled by calculating a detection $p$ value defined as the proportion of samples reporting background signal levels for both methylated and unmethylated channels. The detection $p$ was calculated using the free $\mathrm{R}$ package minfi (http://bioconductor.org/ packages/release/bioc/html/minfi.html). Probes with detection $p>0.05$ were dropped from subsequent analysis. In addition, we also removed CpG probes harbouring SNPs considering potential disruption on their methylation levels by heterozygous SNPs. As usual, we also dropped methylation data on sex chromosomes to focus on autosomal CpG sites. A total of $473,864 \mathrm{CpGs}$ were available for subsequent analysis. Data normalization was done using the subset-quantile within-array normalization (SWAN) [18] implemented in minfi. At each CpG site, the DNA methylation level was summarized by calculating a "beta" value defined by the Illumina's formula as $\beta=\mathrm{M} /(\mathrm{M}+\mathrm{U}+100)$, where $\mathrm{M}$ and $\mathrm{U}$ are methylated $(\mathrm{M})$ and unmethylated $(\mathrm{U})$ signal intensities measured at the $\mathrm{CpG}$ site. We further removed CpGs with $\beta<0.05$ [19] leaving 427,555 CpGs for statistical analysis. Both raw and processed DNA methylation data for the discovery samples have been deposited to the NCBI GEO database (http://www.ncbi.nlm.nih.gov/geo) under accession number GSE61496.

\section{The replication samples}

The replication sample was comprised of 175 pairs of identical twins (350 individuals, 192 males and $158 \mathrm{fe}-$ males) aged 56 to 80 years with a median age of 66 (Table 1). The estimated weeks before term birth were provided by midwives with a range from 0 to 8 weeks. Preterm birth was defined as birth at least 3 weeks before term. A total of 40 twins were found as preterm births. Blood sampling, DNA extraction and methylation analysis were performed as described for the discovery samples. The methylation data were also normalized using the Subset-quantile Within Array Normalization (SWAN) [18]. Methylation "beta" values were likewise calculated using the Illumina's formula.

\section{Estimating and adjusting cell composition}

Cell composition in whole blood can change with age. Although the age range of our discovery samples was relatively short, that of the replication samples was 24 years (Table 1). For the discovery samples, we controlled for this issue by estimating and correcting for cell composition in each individual. We estimated cell composition for six blood cell types: CD8T, CD4T, natural killer cell, B cell, monocyte and granulocyte based on our measured DNA methylation data from whole blood using an approach proposed by Houseman et al. [20], using the $\mathrm{R}$ package minfi. For the replication samples, blood leukocyte subtypes (monocytes, lymphocytes, basophils, neutrophils and eosinophiles), counted using a Coulter LH 750 Haematology Analyser, were available. Missing blood cell counts were imputed by a modified version of the method supplied by PredictCellComposition (www.github.com/mvaniterson/predictcellcomposition). The effect of cell composition was then adjusted for by including the estimated proportion of each cell type as covariates in the regression analyses.

\section{Data analysis}

The association between DNA methylation and PTB was investigated on both single CpGs and genomic regions through fitting linear models that regressed the level of DNA methylation on PTB status adjusting for sex, birthweight and cell compositions. Before fitting the models, DNA methylation $\beta$-values were transformed into $M$ values using logit transformation to ensure normal or approximately normal distribution.

\section{Single-CpG-based analysis}

We applied a linear regression model with a robust sandwich variance estimator to regress the methylation $M$ values on PTB status (preterm coded as 1 and term coded as 0), sex, birthweight and estimated cell compositions. The sandwich variance estimator was introduced to take into accounts the intra-pair twin correlation on DNA methylation. By estimating and testing the regression coefficient for PTB, we were interested in identifying differentially methylated CpG probes (DMPs) of PTB. The model was fitted using the clubSandwich package in R (https:// cran.r-project.org/web/packages/clubSandwich). $P$ values were adjusted for multiple testing by calculating the false discovery rate [21] (FDR) with genome-wide significance defined as FDR $<0.05$.

\section{Multiple-CpG-based analysis}

On top of the single-CpG-based analysis, we further extended our analysis to multiple CpGs to look for differentially methylated genomic regions (DMRs) in association with PTB. This was done using the bumphunter approach introduced by Jaffe et al. [22] implemented in the $\mathrm{R}$ package minfi. The methylation $M$ values were first regressed on sex, birthweight and estimated cell compositions. The residuals from the regression and PTB status were then submitted to the bumphunter() function in minfi. The approach assumes that the locus-specific estimates of regression coefficients $(\beta s)$ are smooth along the strand of a chromosome and applies the loess smoothing technique to smooth coefficient $\beta$ s within a pre-defined chromosomal region (300 base pairs in our analysis). After smoothing, the 99th percentile of the smoothed $\beta \mathrm{s}$ can be calculated to obtain upper and lower thresholds. These thresholds 
are then used to define hyper- or hypo-methylated DMRs with smoothed peaks above or below the thresholds. For each DMR identified, a sum statistic is calculated by taking the sum of the absolute values of all the smoothed $\beta \mathrm{s}$ within that region. The sum statistic is then used to rank all DMRs with the top-most important DMR having the highest sum statistic value. Statistical significance of the DMRs is assessed by computer permutation (we set 1000 replications) in combination with correction for multiple testing to obtain family-wised error rate (FWER) [22].

\section{Biological pathway analysis}

Advantaged by the multiple-CpG-based analysis that outputs genomic locations of the identified DMRs, biological pathway analysis was conducted by submitting the chromosomal coordinates of the detected DMRs to the Genomic Regions Enrichment of Annotations Tool (GREAT) at http://bejerano.stanford.edu/great/public/html/ to analyse the functional significance of cis-regulatory regions identified by localized measurements of DNA binding events across an entire genome [23] using the Genome Reference Consortium Human Build 37 (GRCh37) as the RefSeq database. GREAT incorporates annotations from 20 ontologies and associates genomic regions with genes by defining a 'regulatory domain' for each gene such that all non-coding sequences that lie within the regulatory domain are assumed to regulate that gene. The 'two nearest genes' was assigned as the association rule from genomic regions to genes, which extends each gene's regulatory domain from its transcription start site (TSS) to the nearest upstream and downstream TSS, up to $1 \mathrm{MB}$ in each direction. Both the binomial test over genomic regions and the hypergeometric test over genes were performed to provide an accurate picture of annotation enrichments [23].

\section{Genomic plotting}

Visualization and annotation of genomic segments hosting regions under differential methylation were realized by integrative plotting using $R$ package Gviz [24]. Information on genomic annotation was taken from the UCSC hg19 assembly.

\section{Results}

\section{Discovery EWAS}

We first performed an EWAS on the 144 discovery samples using regression analysis on each of the 473,864 CpGs after filtering, measured using the Illumina Infinium HumanMethylation450 Beadchip assay (see the 'Methods' section for details). From the volcano plot (Fig. 2) and Manhattan plot (Additional file 1: Figure $\mathrm{S} 1$ ), it can be seen that no CpG reached genome-wide significance level of FDR $<0.05$ for the effect of PTB (Additional file 2: Table S1). We continued our discovery

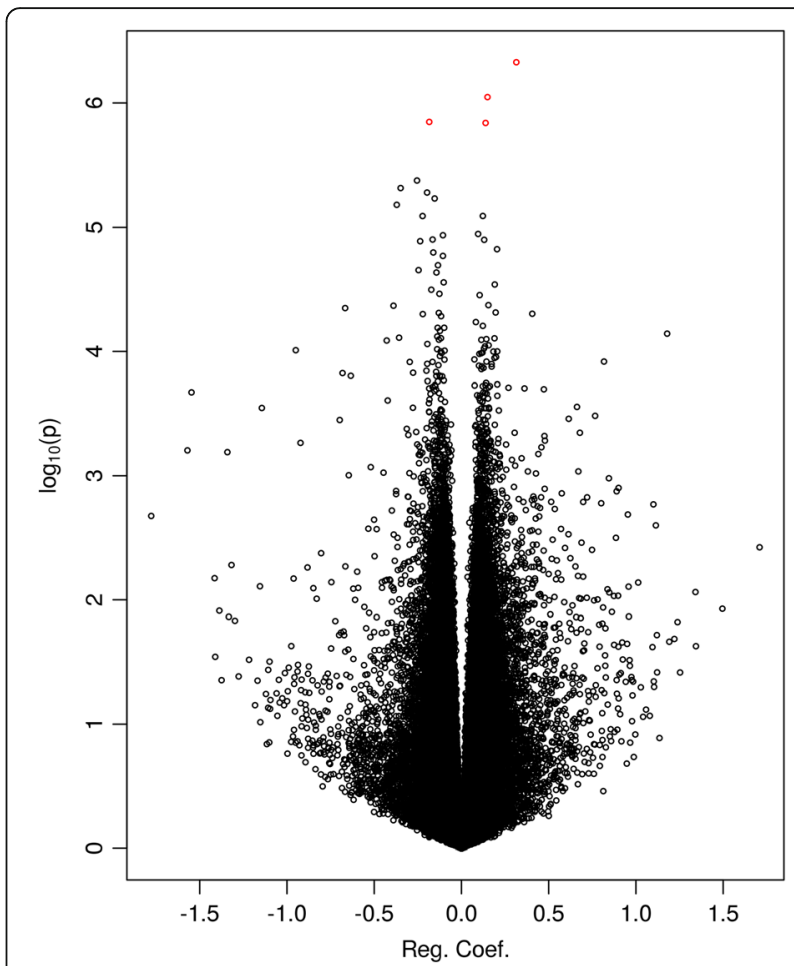

Fig. 2 Volcano plot displaying minus log scaled $p$ value with base 10 from single CpG site-based EWAS plotted against regression coefficient of PTB. The top red dots are four CpGs with $0.1<\mathrm{FDR}<0.2$

EWAS by performing genomic region-based analysis using the bumphunter function in the free $\mathrm{R}$ package minfi (see the 'Methods' section for detail). By focusing on regions with a mean methylation difference of over $10 \%$ between PTB and term births, we found a list of 16,508 regions (Additional file 3: Figure S2) and among them 2651 regions with $p$ value $<0.05$ (Additional file 4: Table S2). Table 2 shows the top six regions with FWER $<0.1$, three of them with FWER $<0.05$. Among the top three DMRs, the most significant was annotated to the promotor region of SDHAP3 gene on chromosome 5 at p15.33 exhibiting a clear pattern of hypomethylation (Figs. 3a and 4a); the second most significant DMR was hypermethylated in the gene body (second or third intron) of TAGLN3 on chromosome 3 at q13.2 (Figs. 3b and $4 \mathrm{~b}$ ); and the third DMR was hypermethylated in the promotor region of the GSTT1 gene on chromosome 22 at q11.23 (Figs. 3c and 4c). Of the three less significant DMRs with FWER <0.1, two were hypomethylated in the promotor region of the DUSP22 and NFYA/ LOC221442 genes on chromosome 6 (Fig. 5a, b), and one was hypermethylated in the promotor of mir886 on chromosome 5 (Fig. 5c). In Additional file 5: Table S3, we show the detailed information on statistical estimate and biological annotations for single CpGs in each of the DMRs in Figs. 3, 4 and 5 (Table 2). CpGs in each DMR 
Table 2 Characterizations of the six identified DMRs with FWER $<0.1$

\begin{tabular}{|c|c|c|c|c|c|c|c|c|c|c|c|}
\hline \multirow[t]{2}{*}{ DMR } & \multirow[t]{2}{*}{ Chr } & \multirow[t]{2}{*}{ Start } & \multirow[t]{2}{*}{ End } & \multicolumn{3}{|c|}{ Discovery } & \multicolumn{2}{|c|}{ Replication } & \multirow[b]{2}{*}{ Linked genes } & \multirow[b]{2}{*}{ Gene region } & \multirow[b]{2}{*}{ Location to CGI } \\
\hline & & & & Value & $p$ value & FWER & Value & $p$ value & & & \\
\hline 1 & 5 & $1,594,282$ & $1,594,863$ & -0.505 & $4.46 \mathrm{E}-07$ & 0.01 & -0.157 & $1.90 \mathrm{E}-04$ & SDHAP3 & $\begin{array}{l}\text { Body, TSS200, } \\
\text { TSS1500 }\end{array}$ & Island \\
\hline 2 & 3 & $111,730,545$ & $111,730,545$ & 1.571 & $7.92 \mathrm{E}-07$ & 0.02 & 0.891 & $8.88 \mathrm{E}-05$ & TAGLN3 & Body & NA \\
\hline 3 & 22 & $24,384,159$ & $24,384,573$ & 0.455 & $2.28 \mathrm{E}-06$ & 0.04 & 0.184 & 5.03E-04 & GSTT1 & 1stExon, 5'UTR, TSS200 & Island \\
\hline 4 & 6 & 291,687 & 292,596 & -0.453 & 4.26E-06 & 0.08 & 0.311 & $2.55 E-05$ & DUSP22 & $\begin{array}{l}\text { 1stExon, 5'UTR, } \\
\text { TSS200, TSS1500 }\end{array}$ & Island, N-Shore \\
\hline 5 & 6 & $41,068,646$ & $41,068,752$ & -0.512 & 4.46E-06 & 0.08 & 0.382 & $1.70 \mathrm{E}-05$ & $\begin{array}{l}\text { NFYA, } \\
\text { LOC221442 }\end{array}$ & $3^{\prime} U T R$ & Island \\
\hline 6 & 5 & $135,415,258$ & $135,416,613$ & 0.271 & 4.90E-06 & 0.10 & 0.197 & $8.68 \mathrm{E}-06$ & MIR886 & $\begin{array}{l}\text { Body, TSS200, } \\
\text { TSS1500 }\end{array}$ & $\begin{array}{l}\text { Island, N-Shore, } \\
\text { S-Shore }\end{array}$ \\
\hline
\end{tabular}

show similar direction of effect and tend to have low nominal $p$ values that may, however, not reach statistical significance individually.

\section{Functional analysis of significant DMRs}

To study the biological functions of the 2651 regions with $p<0.05$ (Additiona file 5: Table S2), we used the online annotation tool GREAT (see the 'Methods' section) for exploring the cis regulatory regions of nearby genes, making use of the Molecular Signatures Database (MSigDB) which is one of the most widely used and comprehensive databases of gene sets for performing gene set enrichment analysis. The analysis identified five MSigDB pathways, all involved in immune responses
(Table 3). Both the binomial test over genomic regions and the hypergeometric test over genes showed very high significance for the five pathways as indicated by their FDRs $(F D R<8.13 e-28$ for binomial test; FDR $<9.37 \mathrm{e}-03$ for hypergeometric test).

\section{Replication of top significant DMRs}

Using the old twin samples, we performed independent replication analysis of the six DMRs in Table 2. For each DMR, the same set of CpGs as in the discovery stage was selected according to genomic location. Differential methylation between PTB and term birth replication samples was estimated in the same manner as for the discovery analysis (adjusting for age, sex and cell

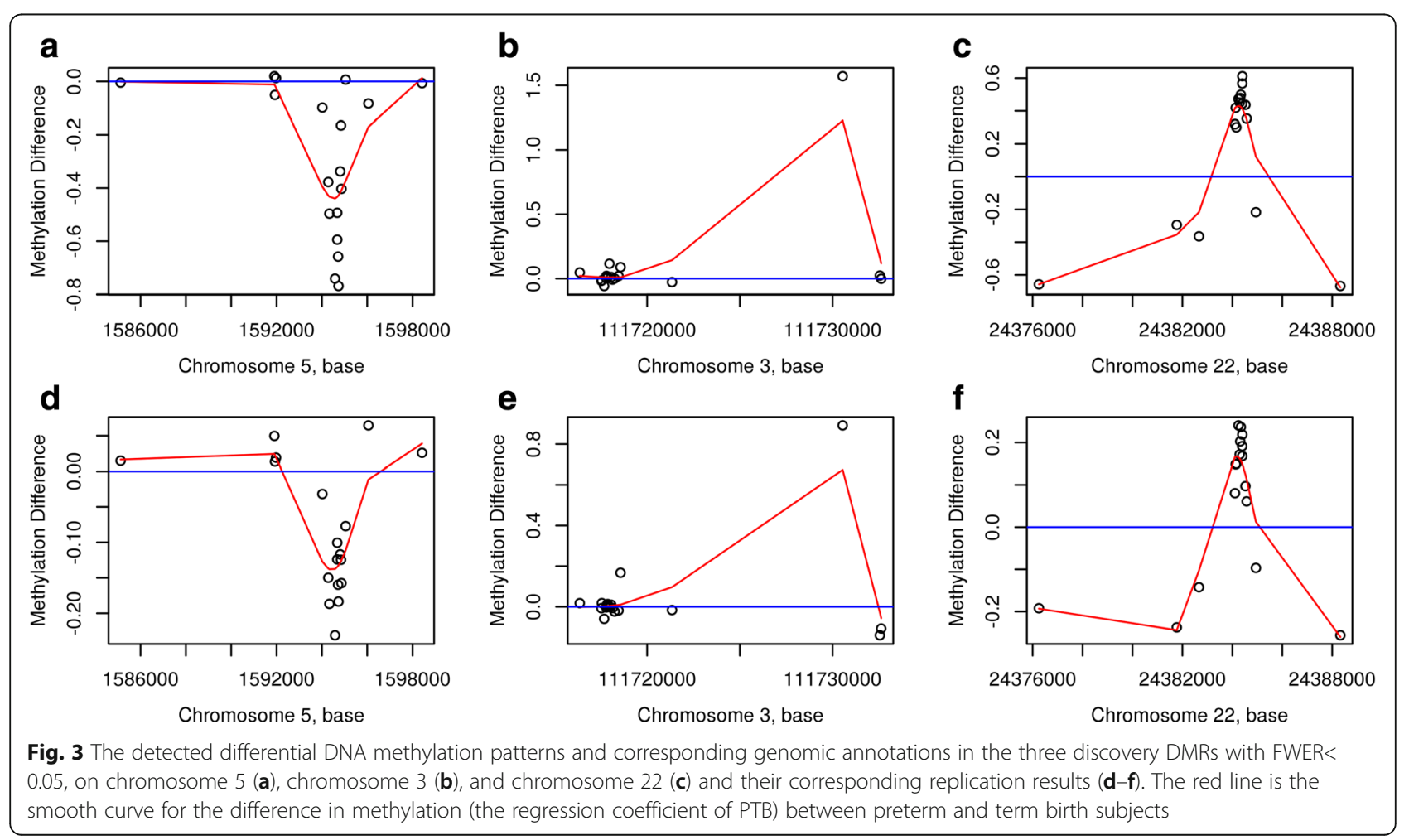




\section{a}
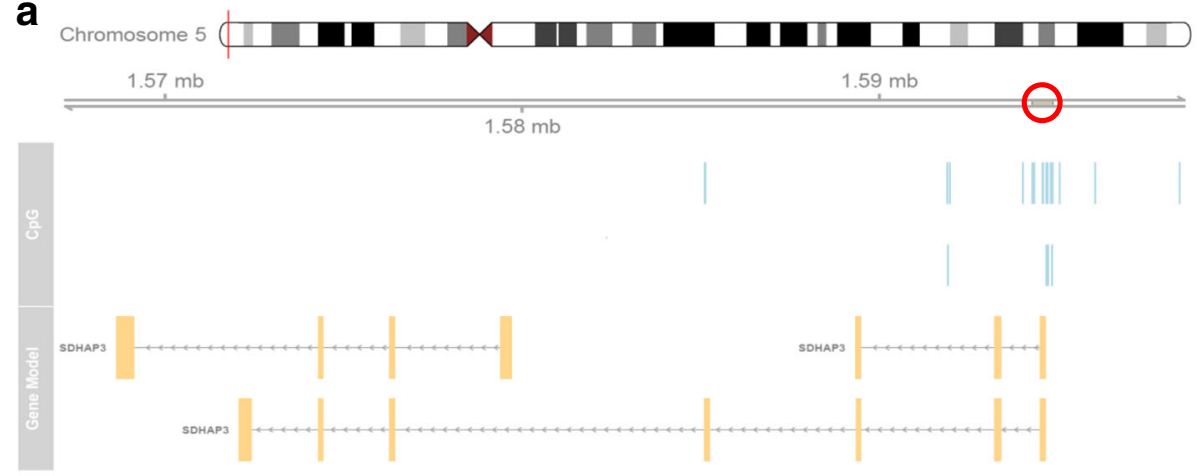

b

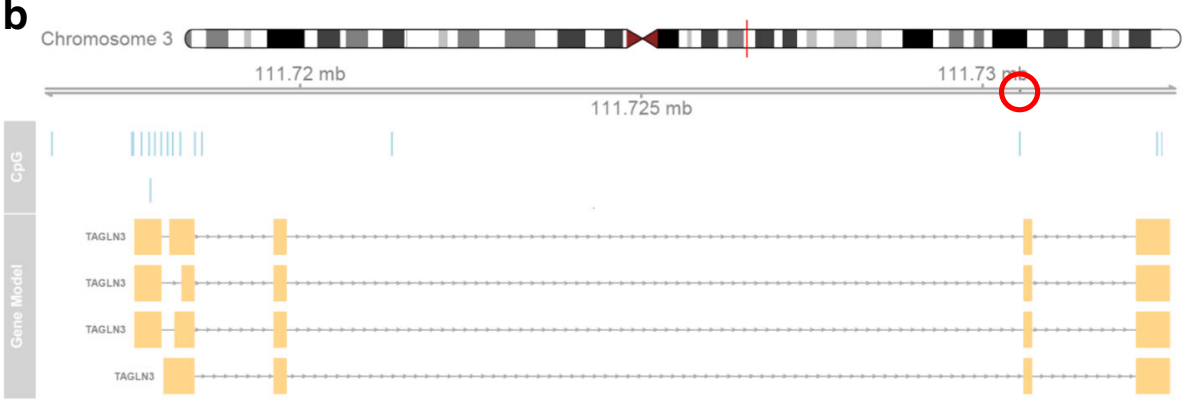

C
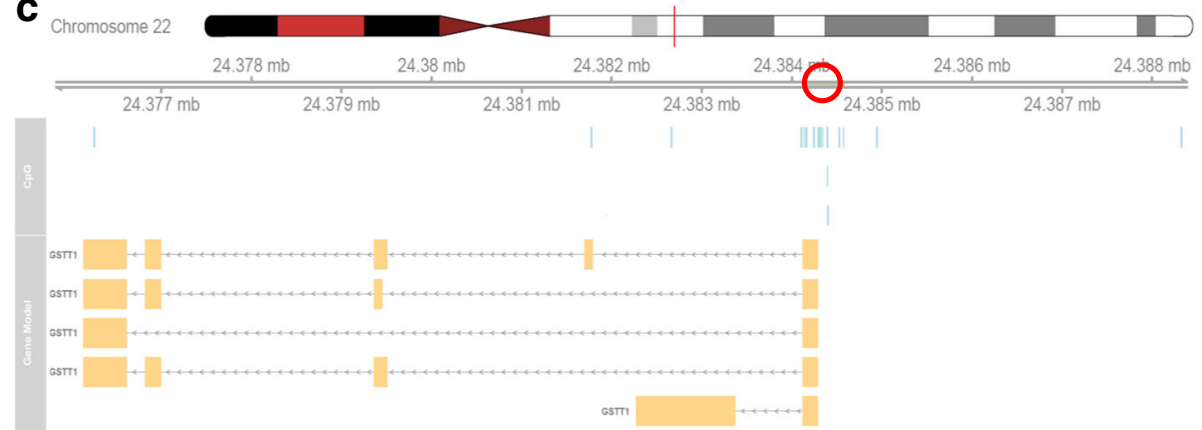

Fig. 4 Genomic regions hosting the significant discovery DMRs (indicated by the red circles) on chromosomes 5 (a), 3 (b) and 22 (c) plotted against the CpG sites in Fig. 3 and corresponding gene annotations

composition). Figure $3 \mathrm{~d}-\mathrm{f}$ displays the replicated differential methylation patterns corresponding to the three discovery-stage DMRs in Fig. 3a-c. As shown in Fig. 3, the three DMRs with FWER $<0.05$ were all nicely replicated with nearly the same patterns. Table 2 shows very low nominal $p$ values for the replication DMRs: $1.9 \mathrm{e}-04$, $8.88 \mathrm{e}-05$ and $5.03 \mathrm{e}-04$ respectively.

In Fig. $5 \mathrm{~d}-\mathrm{f}$, we show the replication results for the three less significant discovery DMRs with $0.05<$ FWER $<$ 0.1 . Interestingly, although a similar methylation pattern was replicated for the DMR on chromosome 5, the other two DMRs on chromosome 6 were replicated with again similar patterns but opposite directions. The corresponding patterns in the replication samples were not random patterns considering their very low nominal $p$ values (2.55e-05, 1.7e-05 and 8.68e-06 respectively) (Table 2 ).

\section{Discussion}

We have performed the first genome-wide association study on the epigenetic effect of preterm birth in adults. Cruickshank et al. [14] investigated PTB-related epigenetic changes at birth and at 18 years of age and reported no genome-wide significant finding in their samples from 18-year-olds. Likewise, our analysis did not identify any $\mathrm{CpG}$ sites reaching genome-wide significance in the discovery samples of young adults. The highly valuable findings in this study come from genomic region-based association analysis that jointly tested the association of 


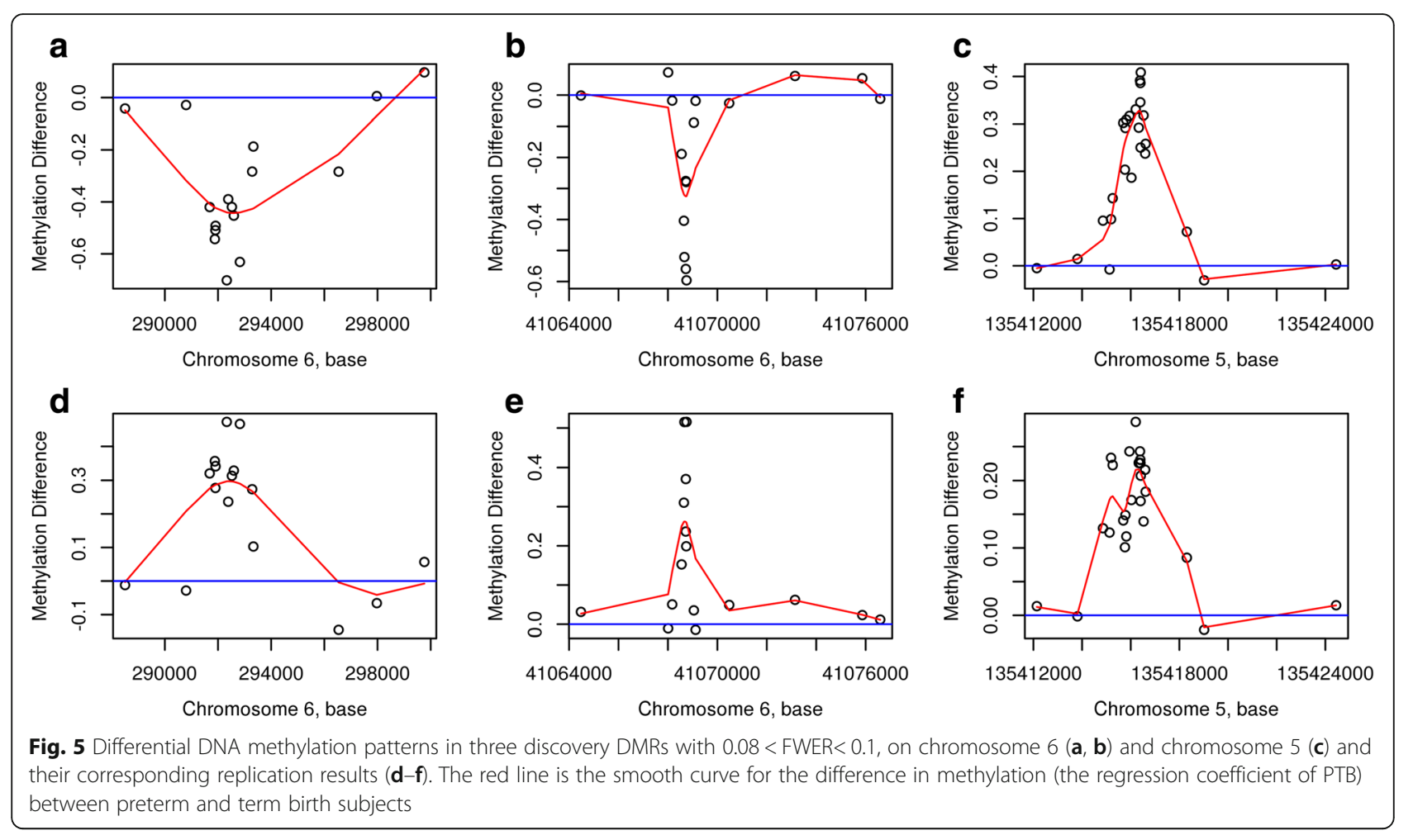

groups of adjacent CpGs that form DMRs. As shown in Table 2, multiple genomic regions were found as differentially methylated in association with PTB. The results indicate that, as an early life event, PTB could impose differential epigenetic patterns that can be detected in the DNA methylome of adult subjects in their thirties as in the discovery samples and even at old ages as in the replication samples.

Among the genes linked to the most significant DMRs in Table 2, SDHAP3 has very recently been implicated in smoking, as significantly decreased methylation at the CpG island within the promoter region of SDHAP3 on chromosome 5 was reported in smoking-exposed foetuses [25]. The PTB associated methylation pattern as shown in Figs. 3a and 4a points to the same direction and genomic location although maternal smoking information is not available in our study. A differential DNA methylation pattern was also found in SDHAP3 when comparing autistic brains and control [26]. The second most significant DMR is in the gene body of TAGLN3 (Fig. 4b). This gene (also known as NP22, encoding a novel cytoskeleton-associated protein) is differentially expressed in human alcoholic brain [27] and in the anterior cingulate cortex of schizophrenia [28].

Perhaps, the most interesting DMR found in this study is the third DMR in Table 2. This DMR sits in the promotor region of GSTT1 (glutathione $S$-transferases gene theta 1) on chromosome 22 (Fig. 4c). Polymorphisms in the GST genes are partially responsible for the variability in GST enzymatic activity across individuals. Maternal genetic variations (the null genotype or homozygous deletion) in GSTT1 have been intensively associated with an increased risk of preterm delivery and low birthweight, alone $[29,30]$ or in combination with smoking

Table 3 MSigDB Pathways enriched by DMRs with nominal $p<0.05$ from GREAT analysis

\begin{tabular}{|c|c|c|c|c|c|c|c|c|c|c|c|}
\hline \multicolumn{6}{|l|}{ Binomial model } & \multicolumn{6}{|c|}{ Hypergeometric model } \\
\hline Term Name & Rawp value & $F D R$ & $\begin{array}{l}\text { Fold } \\
\text { enrichment }\end{array}$ & Exp. & $\begin{array}{l}\text { Obs. } \\
\text { Gene Hits }\end{array}$ & Rawp value & $F D R$ & $\begin{array}{l}\text { Fold } \\
\text { enrichment }\end{array}$ & Exp. & $\begin{array}{l}\text { Obs.Gene } \\
\text { Hits }\end{array}$ & TotalGenes \\
\hline $\begin{array}{l}\text { Antigen processing and } \\
\text { presentation }\end{array}$ & $1.33 e-55$ & $1.76 e-52$ & 12.28 & 6.27 & 77 & $3.55 e-5$ & $9.37 e-3$ & 2.10 & 13.79 & 29 & 80 \\
\hline Graft-versus-host disease & $5.65 e-53$ & $3.73 e-50$ & 14.46 & 4.63 & 67 & $2.17 e-6$ & $9.56 \mathrm{e}-4$ & 2.98 & 6.38 & 19 & 37 \\
\hline Type I diabetes mellitus & $1.52 \mathrm{e}-50$ & $6.72 \mathrm{e}-48$ & 10.20 & 7.65 & 78 & $1.24 \mathrm{e}-7$ & $1.63 e-4$ & 3.11 & 7.07 & 22 & 41 \\
\hline Allograft rejection & $7.25 e-50$ & $2.40 e-47$ & 12.55 & 5.42 & 68 & $7.10 \mathrm{e}-7$ & $4.69 e-4$ & 3.15 & 6.03 & 19 & 35 \\
\hline Viral myocarditis & $4.92 \mathrm{e}-30$ & $8.13 e-28$ & 5.13 & 15.02 & 77 & $9.64 \mathrm{e}-6$ & $3.18 \mathrm{e}-3$ & 2.30 & 11.72 & 27 & 68 \\
\hline
\end{tabular}


[31-33]. The interaction between GSTs and smoking shows the involvement of epigenetic mechanism that links maternal behaviour and genetic susceptibility in contributing to adverse pregnancy outcomes. The association between GST genetic variation and PTB has been observed not only in the mother but also in the child [34]. In fact, Bustamante et al. [35] found that the child genotype is responsible for the effect after adjusting for maternal genotype. As our observation is based on PTB adults, our result is in line with their conclusion but from an epigenetic perspective. Most importantly, the latter suggests that environmental factors could also be involved in the association between GSTT1 and PTB through the epigenetic mechanism. Taken together, both genetic and epigenetic variations in the child can be associated with PTB. The coherence between genetics and epigenetics here is sensible because DNA methylation at the promotor region turns the gene off, which is equivalent to a deletion or the null genotype of the gene.

As an extra effort, we explored the transcriptional profiles of the genes linked to significant DMRs. Gene expression data on two genes, TAGLN3 and GSTT1, were available from the Agilent Human Gene Expression Microarray (v3) applied to the same discovery samples. After adjusting for covariates, no expression difference was found for TAGLN3 between term birth and PTB ( $p$ $=0.639)$ while a borderline significance $(p=0.059)$ for the down-expression of GSTT1 in PTB (Additional file 6: Figure S3). Although the expression of GSTT1 can also be regulated by other mechanisms or influenced by deletion of the gene in PTB subjects, the reduced expression level in PTB group provides alternative evidence in support of DNA methylation analysis.

Among the three less significant DMRs in Table 2, the last one on chromosome 5 is replicated by a similar pattern in the old twins (Fig. 5f). The CpGs in this region are hypermethylated in the promotor region and gene body of mir886, a noncoding RNA repressed in cancer [36, 37]. The two DMRs on chromosome 6 display significant patterns ( $p$ values $2.55 \mathrm{e}-05$ and $1.70 \mathrm{e}-05$ ) in the replication samples but with opposite directions as compared to the discovery DMRs (hypomethylation in the discovery samples, and hypermethylation in the replication samples) which could possibly suggest age-dependent effects. The DMR located on chromosome 6 from bp 291,687 to 292,596 covers the promotor region of the DUSP22 gene. Epigenetic alteration of this gene has been shown to mediate Alzheimer's disease [38] and dementia [39]. Interestingly, hypomethylation of the DUSP22 promotor has been reported to correlate with duration of service in firefighters [40]. The observed epigenetic modification could result from exposure to complex mixtures of toxic substances from burning and overheated materials. Although the smoking status of mothers of our twins is not available, the finding among the firefighters could resemble smoking-exposed foetuses. The DMR located from bp $41,068,646$ to $41,068,752$ on chromosome 6 is at the $3^{\prime}$ UTR of the NFYA (nuclear transcription factor Y) gene. As a transcription factor, NFYA binds to the CAAT box in promotors of many genes in eukaryotes and functions as a regulator of their overexpression in several types of cancer [41]. Note that the same gene has been found to be persistently hypermethylated by PTB in an epigenome-wide association study on both newborn and 18-year-old samples [14]. In brief, the genes covered by these less significant DMRs are implicated in neurodegenerative disorders and risk of cancer as well.

PTB newborns have immature immune systems with reduced innate and adaptive immune function [42]. It is interesting to see that four of the five pathways in Table 3 overlap with pathways deduced from genes linked to CpGs showing significant correlation in maternal and PTB fetal methylation [43], and that all the five pathways appeared in the enriched functional pathways from genes with copy number variations in common miscarriage [44]. Results from our biological pathway analysis reconfirm the importance of the immune system in PTB but in adult samples. Meanwhile the overlap in biological pathways could also suggest the broad involvement of immunity in labour complications in general. Most importantly, the immune implication of PTB could persist into adult life and even old ages. The high involvement of the immune system in PTB as revealed by region-based analysis can also be seen from the Manhattan plot for DMRs (Additional file 3: Figure S2) when compared with the Manhattan plot for single-CpG sites (Additional file 1: Figure S1). The former displays a clearer enrichment pattern of DMRs in the major histocompatibility complex (MHC) region on chromosome 6.

The fact that the significant DMRs were identified and replicated in independent and much older samples has a twofold significance. First, it reveals functional genes differentially regulated in association with PTB through epigenetic mechanism; the latter could serve to link PTB with maternal environmental exposure or lifestyle factors to provide clue for prevention of PTB. Second, and also most importantly, the altered DNA methylation patterns observed in our discovery young adults persist in old subjects of up to 80 years of age, suggesting that some of the PTB-associated epigenetic modifications can be long-lasting or perhaps persistent throughout the entire life. In summary, our genomic region-based analysis of the DNA methylome identified epigenetic fingerprints of premature birth in young adult subjects, consistently replicable in old adults. Functional annotation of the significant methylation patterns associated with PTB revealed genes involved in adverse pregnancy outcomes, in neurodevelopmental disorders and in cancer 
susceptibility, providing epigenetic evidence of long-term effects of early life events in support of the developmental origin of disease and health.

Finally, it should be kept in mind that our significant findings are based on twins. Even though findings from this study are highly relevant to PTB in general (e.g. the GSTT1 gene), generalization of our results should be done with caution because the aetiology of PTB in twins could involve risk factors specific for twin pregnancies [45], such as uterine overdistention [46]. Further replication studies using twin and non-twin samples are warranted for validation, justification and generalization of our findings.

\section{Conclusions}

This study provides novel evidence for PTB-associated epigenetic regulation in important genes/pathways and meanwhile reveals that premature delivery, as an early life event, could be related to differential methylation regulation patterns observable in adults and even at high ages which could potentially mediate susceptibility to age-related diseases and adult health.

\section{Additional files}

Additional file 1: Figure S1. Manhattan plot for EWAS results based on single CpG sites on autosomal chromosomes. The $y$-axis shows the negative log-base-10 of the $P$ value for each CpG. (PNG $1276 \mathrm{~kb}$ )

Additional file 2: Table S1. PTB EWAS results with $p$ value below $1 \%$. (PDF $570 \mathrm{~kb}$ )

Additional file 3: Figure S2. Manhattan plot for genomic regions from region based analysis on autosomal chromosomes. The $y$-axis shows the negative log-base-10 of the $p$ value for each genomic region. (PNG $932 \mathrm{~kb}$ )

Additional file 4: Table S2. PTB DMR with $p$ value lower than $5 \%$. (PDF $991 \mathrm{~kb}$ )

Additional file 5: Table S3. CpGs under top DMRs. (PDF $71 \mathrm{~kb}$ )

Additional file 6: Figure S3. Boxplot displaying the expression patterns of GSTT1 gene in preterm (PTB) and term (TB) groups. The gene shows reduced expression level in the PTB group. (PNG $87 \mathrm{~kb}$ )

\section{Abbreviations \\ CpG: Cytosine-phosphate-guanine; DMBR: Danish medical birth registry; DMP: Differentially methylated probe; DMR: Differentially methylated region; EDTA: Ethylene di-amine tetra acetic acid; EWAS: Epigenome-wide association study; FDR: False discovery rate; FWER: Family-wise error rate; GREAT: Genomic Regions Enrichment of Annotations Tool; MHC: Major histocompatibility complex; MSigDB: Molecular Signatures Database; SWAN: Subset-quantile Within Array Normalization; TSS: Transcription start site(s)}

\section{Acknowledgements}

This study was supported by the Integrated research on Developmental determinants of Aging and Longevity (IDEAL), EU's FP7 project number: 259679 and by the DFF research project 1 from the Danish Council for Independent Research, Medical Sciences (DFF-FSS), project number: DFF 6110-00114. The work of Shuxia Li was jointly supported by the Velux Foundation research grant number 000121540 and Novo Nordisk Foundation Medical and Natural Sciences Research Grant [grant number NNF130C0007493].

\section{Availability of data and materials}

Both raw and processed DNA methylation data have been deposited to the NCBI GEO database http://www.ncbi.nlm.nih.gov/geo/ under accession number GSE61496.

\section{Authors' contributions}

QT, LC, KC and MF contributed to the conception and design. SL, QT, LC, MN, MS and ML contributed to the analysis and interpretation. QT and SL contributed to the drafting of the manuscript. All authors read and approved the final manuscript.

\section{Ethics approval and consent to participate}

Informed consent was obtained for all participants; the study was approved by The Regional Scientific Ethical Committees for Southern Denmark (S20090033) and conducted in accordance with the Helsinki II declaration.

\section{Consent for publication}

Informed written consent was obtained from all the participants. Additionally, the manuscript does not contain any individual person's data in any form.

\section{Competing interests}

The authors declare that they have no competing interests in this work.

\section{Publisher's Note}

Springer Nature remains neutral with regard to jurisdictional claims in published maps and institutional affiliations.

\section{Author details}

${ }^{1}$ Epidemiology and Biostatistics, Department of Public Health, Faculty of Health Science, University of Southern Denmark, J. B. Winsløws Vej 9B, DK-5000 Odense, Denmark. ²Unit of Human Genetics, Department of Clinical Research, University of Southern Denmark, Odense, Denmark. ${ }^{3}$ Department of Endocrinology, Odense University Hospital, Odense, Denmark. ${ }^{4}$ Department of Clinical Genetics, Odense University Hospital, Odense, Denmark.

Received: 28 February 2018 Accepted: 11 June 2018

Published online: 27 June 2018

\section{References}

1. Chauhan SP, Scardo JA, Hayes E, Abuhamad AZ, Berghella V. Twins: prevalence, problems, and preterm births. Am J Obstet Gynecol. 2010; 203(4):305-15.

2. Luu TM, Katz SL, Leeson P, Thébaud B, Nuyt AM. Preterm birth: risk factor for early-onset chronic diseases. CMAJ. 2016;188:736-46.

3. Kerkhof GF, Breukhoven PE, Leunissen RW, Willemsen RH, Hokken-Koelega AC. Does preterm birth influence cardiovascular risk in early adulthood? J Pediatr. 2012;161:390-6.

4. Parkinson JR, Hyde MJ, Gale C, Santhakumaran S, Modi N. Preterm birth and the metabolic syndrome in adult life: a systematic review and meta-analysis. Pediatrics. 2013;131(4):e1240-63.

5. Fraser J, Walls M, McGuire W. Respiratory complications of preterm birth. BMJ. 2004;329:962-5.

6. Abbott A. Babies are increasingly surviving premature birth-but researchers are only beginning to understand the lasting consequences for their mental development. Nature. 2015;518:24-6.

7. Perera F, Herbstman J. Prenatal environmental exposures, epigenetics, and disease. Reprod Toxicol. 2011:31:363-73.

8. Ventura-Junca R, Herrera LM. Epigenetic alterations related to early-life stressful events. Acta Neuropsychiatr. 2012;24:255-65.

9. Vaiserman AM. Epigenetic programming by early-life stress: evidence from human populations. Dev Dyn. 2015;244:254-65.

10. Menon R, Conneely KN, Smith AK. DNA methylation: an epigenetic risk factor in preterm birth. Reprod Sci. 2012;19(1):6-13.

11. Maddalena P. Long term outcomes of preterm birth: the role of epigenetics. Newborn Infant Nurs Rev. 2013;13:137-9.

12. Parets SE, Bedient CE, Menon R, Smith AK. Preterm birth and its long-term effects: methylation to mechanisms. Biology (Basel). 2014;3:498-513.

13. Schroeder JW, Conneely KN, Cubells JC, Kilaru V, Newport DJ, Knight BT, Stowe ZN, Brennan PA, Krushkal J, Tylavsky FA, Taylor RN, Adkins RM, Smith 
AK. Neonatal DNA methylation patterns associate with gestational age. Epigenetics. 2011;6:1498-504.

14. Cruickshank MN, Oshlack A, Theda C, Davis PG, Martino D, Sheehan P, Dai Y, Saffery R, Doyle LW, Craig JM. Analysis of epigenetic changes in survivors of preterm birth reveals the effect of gestational age and evidence for a longterm legacy. Genome Med. 2013;5(10):96.

15. Simpkin AJ, Suderman M, Gaunt TR, Lyttleton O, McArdle WL, Ring SM, Tilling K, Davey Smith G, Relton CL. Longitudinal analysis of DNA methylation associated with birth weight and gestational age. Hum Mol Genet. 2015;24:3752-63.

16. Sparrow S, Manning JR, Cartier J, Anblagan D, Bastin ME, Piyasena C, Pataky R, Moore EJ, Semple SI, Wilkinson AG, Evans M, Drake AJ, Boardman JP. Epigenomic profiling of preterm infants reveals DNA methylation differences at sites associated with neural function. Transl Psychiatry. 2016;6:e716.

17. Tan Q, Frost M, Heijmans BT, von Bornemann Hjelmborg J, Tobi EW, Christensen K, Christiansen L. Epigenetic signature of birth weight discordance in adult twins. BMC Genomics. 2014;15:1062.

18. Maksimovic J, Gordon L, Oshlack A. SWAN: subset-quantile within array normalization for illumina infinium HumanMethylation450 BeadChips. Genome Biol. 2012;13(6):R44.

19. Logue MW, Smith AK, Wolf EJ, Maniates H, Stone A, Schichman SA, McGlinchey RE, Milberg W, Miller MW. The correlation of methylation levels measured using Illumina 450K and EPIC BeadChips in blood samples. Epigenomics. 2017;9(11):1363-71.

20. Houseman EA, Accomando WP, Koestler DC, Christensen BC, Marsit CJ, Nelson HH, Wiencke JK, Kelsey KT. DNA methylation arrays as surrogate measures of cell mixture distribution. BMC Bioinformatics. 2012;13:86.

21. Benjamini $Y$, Hochberg Y. Controlling the false discovery rate: a practical and powerful approach to multiple testing. J R Statist Soc B. 1995:57:289-300.

22. Jaffe $A E$, Murakami $P$, Lee $H$, Leek JT, Fallin MD, Feinberg AP, Irizarry RA. Bump hunting to identify differentially methylated regions in epigenetic epidemiology studies. Int J Epidemiol. 2012;41(1):200-9.

23. McLean CY, Bristor D, Hiller M, Clarke SL, Schaar BT, Lowe CB, Wenger AM, Bejerano G. GREAT improves functional interpretation of cis-regulatory regions. Nat Biotechnol. 2010;28:495-501.

24. Hahne F, Ivanek R. Statistical genomics: methods and protocols. In: Mathé E, Davis S, editors. Chapter visualizing genomic data using Gviz and Bioconductor. New York: Springer New York; 2016. p. 335-51.

25. Chatterton Z, Hartley BJ, Seok MH, Mendelev N, Chen S, Milekic M, Rosoklija G, Stankov A, Trencevsja-Ivanovska I, Brennand K, Ge Y, Dwork AJ, Haghighi F. In utero exposure to maternal smoking is associated with DNA methylation alterations and reduced neuronal content in the developing fetal brain. Epigenetics Chromatin. 2017;10:4

26. Ladd-Acosta C, Hansen KD, Briem E, Fallin MD, Kaufmann WE, Feinberg AP. Common DNA methylation alterations in multiple brain regions in autism. Mol Psychiatry. 2014;19:862-71.

27. Fan $L$, Jaquet $V$, Dodd $P R$, Chen W, Wilce PA. Molecular cloning and characterization of hNP22: a gene up-regulated in human alcoholic brain. J Neurochem. 2001;76:1275-81.

28. Ito M, Depaz I, Wilce P, Suzuki T, Niwa S, Matsumoto I. Expression of human neuronal protein 22, a novel cytoskeleton-associated protein, was decreased in the anterior cingulate cortex of schizophrenia. Neurosci Lett. 2005;378:125-30.

29. Parveen F, Faridi RM, Das V, Tripathi G, Agrawal S. Genetic association of phase I and phase II detoxification genes with recurrent miscarriages among north Indian women. Mol Hum Reprod. 2010;16:207-14.

30. Liu Y, Tang YB, Chen J, Huang ZX. Meta-analysis of GSTT1 null genotype and preterm delivery risk. Int J Clin Exp Med. 2014;7:1537-41.

31. Suter M, Abramovici A, Aagaard-Tillery K. Genetic and epigenetic influences associated with intrauterine growth restriction due to in utero tobacco exposure. Pediatr Endocrinol Rev. 2010;8:94-102.

32. Zheng X, Feingold E, Ryckman KK, Shaffer JR, Boyd HA, Feenstra B, Melbye M, Marazita ML, Murray JC, Cuenco KT. Association of maternal CNVs in GSTT1/GSTT2 with smoking, preterm delivery, and low birth weight. Front Genet. 2013;4:196

33. Sheikh IA, Ahmad E, Jamal MS, Rehan M, Assidi M, Tayubi IA, AlBasri SF, Bajouh OS, Turki RF, Abuzenadah AM, Damanhouri GA, Beg MA, Al-Qahtani M. Spontaneous preterm birth and single nucleotide gene polymorphisms: a recent update. BMC Genomics. 2016;17(Suppl 9):759.
34. Nukui T, Day RD, Sims CS, Ness RB, Romkes M. Maternal/newborn GSTT1 null genotype contributes to risk of preterm, low birthweight infants. Pharmacogenetics. 2004;14:569-76.

35. Bustamante M, Danileviciute A, Espinosa A, Gonzalez JR, Subirana I, Cordier S, Chevrier C, Chatzi L, Grazuleviciene R, Sunyer J, Ibarluzea J, Ballester F, Villanueva CM, Nieuwenhuijsen M, Estivill X, Kogevinas M. Influence of fetal glutathione $S$-transferase copy number variants on adverse reproductive outcomes. BJOG. 2012;119:1141-6.

36. Cao J, Song Y, Bi N, Shen J, Liu W, Fan J, Sun G, Tong T, He J, Shi Y, Zhang X, Lu N, He Y, Zhang H, Ma K, Luo X, Lv L, Deng H, Cheng J, Zhu J, Wang L, Zhan Q. DNA methylation-mediated repression of miR-886-3p predicts poor outcome of human small cell lung cancer. Cancer Res. 2013;73:3326-35.

37. Lee K, Kunkeaw N, Jeon SH, Lee I, Johnson BH, Kang GY, Bang JY, Park HS, Leelayuwat C, Lee YS. Precursor miR-886, a novel noncoding RNA repressed in cancer, associates with PKR and modulates its activity. RNA. 2011;17: 1076-89.

38. Sanchez-Mut JV, Aso E, Heyn H, Matsuda T, Bock C, Ferrer I, Esteller M. Promoter hypermethylation of the phosphatase DUSP22 mediates PKAdependent TAU phosphorylation and CREB activation in Alzheimer's disease. Hippocampus. 2014;24:363-8.

39. Delgado-Morales R, Esteller M. Opening up the DNA methylome of dementia. Mol Psychiatry. 2017:22:485-96.

40. Ouyang B, Baxter CS, Lam HM, Yeramaneni S, Levin L, Haynes E, Ho SM. Hypomethylation of dual specificity phosphatase 22 promoter correlates with duration of service in firefighters and is inducible by low-dose benzo[a]pyrene. J Occup Environ Med. 2012:54:774-80.

41. Dolfini D, Mantovani R. Targeting the Y/CCAAT box in cancer: YB-1 (YBX1) or NF-Y? Cell Death Differ. 2013;20:676-85.

42. Melville JM, Moss TJ. The immune consequences of preterm birth. Front Neurosci. 2013;7:79.

43. Parets SE, Conneely KN, Kilaru V, Menon R, Smith AK. DNA methylation provides insight into intergenerational risk for preterm birth in African Americans. Epigenetics. 2015;10:784-92

44. Bagheri H, Mercier E, Qiao Y, Stephenson MD, Rajcan-Separovic E. Genomic characteristics of miscarriage copy number variants. Mol Hum Reprod. 2015; 21:655-61.

45. Aboulghar M, Islam Y. Twin and preterm labor: prediction and treatment Cur Obstet Gynecol Rep. 2013;2:232-9.

46. Campbell S. Universal cervical-length screening and vaginal progesterone prevents early preterm births, reduces neonatal morbidity and is cost saving: doing nothing is no longer an option. Ultrasound Obstet Gynecol. 2011:38(1):1-9.

\section{Ready to submit your research? Choose BMC and benefit from:}

- fast, convenient online submission

- thorough peer review by experienced researchers in your field

- rapid publication on acceptance

- support for research data, including large and complex data types

- gold Open Access which fosters wider collaboration and increased citations

- maximum visibility for your research: over $100 \mathrm{M}$ website views per year

At BMC, research is always in progress.

Learn more biomedcentral.com/submissions 